\title{
Stereo Disparity Calculation in Real-World Scenes with Informative Image Partitioning
}

\author{
N. Brewer ${ }^{1,2}$, N. Liu ${ }^{2}$, L. Wang ${ }^{1}$ \\ ${ }^{1}$ College of Engineering and Computer Science, The Australian National University, Canberra 0200, \\ Australia. \\ ${ }^{2}$ NICTA Canberra Lab, Tower A, 7 London Circuit, Canberra 2600 Australia. \\ Email: nbrewer@cecs.anu.edu.au
}

\begin{abstract}
Despite their presence in almost all real-world navigational environments, finding stereo disparity in large, weakly textured regions is a difficult task. In this paper, we present a method which constructs a novel segmentation of an image into separate planar regions prior to disparity calculation. This segmentation allows us to fit planar models to disparity estimates calculated using a graph cut approach within each segment, producing smooth, accurate disparity maps in ordinarily difficult areas. Our method achieves good results using intensity features in a variety of indoor and outdoor navigational scenes. A comparison of our results with other benchmark approaches is presented, in which the advantages of our technique can be clearly seen.
\end{abstract}

Keywords: Stereo Analysis, Image Segmentation

\section{Introduction}

Using visual information to find the relative or absolute distance of objects in the environment is a challenging problem in computer vision. Depth cues are particularly valuable in navigation tasks, providing information that can allow an agent to successfully avoid obstacles and plan a collisionfree path. Many techniques exist for extracting a depth map from an environment, ranging from dedicated hardware solutions such as laser or sonar range finders to complex probabilistic techniques that infer approximate depth from a single image. Most common techniques used to calculate depth from visual information use multiple cameras to provide sufficient clues as to the distance between the camera pair and objects within the scene. Such techniques generally work by finding a disparity map of the scene, which indicates how much a particular pixel moves between images. At any point, this disparity correlates strongly to the distance between the camera pair and the corresponding object in the image. Many methods exist for finding this disparity, a subset of which are presented in Section 2.

In urban environments, many surfaces and objects are both planar and weakly textured. Examples include road and floor surfaces, to buildings, interior walls and many common large obstacles. In these regions, the distance from a point in the object to the camera changes in a linear fashion, based on the direction in which the surface is oriented relative to the observer. In addition, non-planar surfaces can be broken down into small facets, each of which can be modelled as a plane. This faceting of objects is an underlying assumption of most 3D modelling techniques. Provided the facets are small enough, we are still able to get disparity values which closely resemble the true disparity distribution.

Most navigation tasks take place in these types of multi-planar, real-world environments, and yet many modern stereo disparity methods have difficulty finding good disparity estimates in exactly these scenarios. In this paper, we develop a technique which exploits the natural behaviour of planar surfaces when constructing a disparity map of a scene by making use of Informative Image Partitioning [1]. This enables us to deliver better performance in realistic navigational scenes.

In an Informative Image Partitioning (IIP), the image is partitioned in such a way that 'interesting' objects are divided into a fine partitioning while 'uninteresting' objects have a coarser partitioning. Unlike most segmentation methods, the user interaction involved in IIP generation allows for visually distinct coplanar objects to be readily merged into a single partition, allowing us to avoid the disparity discontinuities that frequently appear at segment boundaries. 
This paper exploits this technique by defining planar, or near planar, objects as uninteresting and more complex objects as interesting. This results in an image partitioned into regions which we are able to assume are planar, as the finer partitioning of non-planar objects will result in the faceting type behaviour described earlier.

By assuming that we have a planar distribution of disparity values within each partition, we are able to fit a plane to disparity values determined using traditional methods to each patch. This produces very smooth results within each partition, allowing us to avoid problems which occur in many methods along large, relatively featureless planar surfaces such as the ground plane. The complete approach taken in this paper is outlined more completely in Section 3.

The algorithm presented in this paper is designed to function best on scenes that are likely to arise in a navigational context. Depth cues are important for navigation, as they provide information on the relative distance between the observer and objects in the environment. In our approach, the initial oversegmentation size is chosen to be quite large, which hinders the performance of the approach in highly non-planar areas. In real-world test images to be used for navigation, this lack of fine disparity resolution does not cause a significant issue.

Our technique shows promising results in many real-world images, both indoor and outdoor, as shown in Section 4. The nature of our algorithm makes it poorly suited to the complex but unrealistic non-navigational environments presented in popular ground-truth data sets, such as Middlebury [2], unsuitable. Generation of suitable images with accurate ground truth was unfeasible for this paper, so we are not able to quantitatively illustrate our technique. Instead, we compare our method to benchmark disparity calculation techniques [3, 4] and show that we achieve significant qualitative improvement in many areas, particularly in low textured planar surfaces.

\section{Related Work}

Finding disparity in stereo image pairs is a major problem of computer vision. There are a large number of methods used for calculating disparity, ranging from simple correlation based pixel matching to sophisticated energy minimisation methods.

The work of Scharstein and Szeliski [2] provides an excellent overview of stereo disparity methods, describing and ranking a number of dense, pixel based stereo matching techniques. Common to the techniques described is an underlying assumption of piecewise smoothness. Numerous recent methods take this assumption a step further, incorporating a segmentation based approach to codify and take advantage of this smoothness.

Many such [5, 6, 7, 8] make use of an unsupervised colour segmentation algorithm, such as mean-shift [9] to generate a set of segments which are then used in a variety of techniques. Bleyer and Gelautz [6] perform explicit segment correspondence and underlying plane detection in addition to utilising segmentation to generate a disparity map. The work of Woodford et al. [10] uses an automated method to segment an image into planar regions by iterated coarse disparity estimation. Using this technique and a second order smoothness prior, they are able to generate high-quality disparity estimates.

Unlike these methods, our approach makes use of an intelligent segmentation method which collects regions belonging to a particular planar segment $a$ priori, see Figure 1. This provides three notable advantages. Firstly, it allows us to fit a single planar model through regions with large visual differences that may otherwise be divided into multiple segments under colour segmentation methods. Secondly, it allows us to assume large scale planar behaviour without intermediate disparity calculation to find regions with similar disparity distributions. Finally, the segmentation approach used is able to maintain a fine partitioning in non-planar areas despite visual similarity, which is difficult for colour segmentation approaches to achieve.

We compare our results to the benchmark disparity calculation methods of Kolmogorov and Zabih [4] and Boykov, Veksler and Zabih [3], together with a sum of absolute differences correlation-based approach based on that provided by with the Bumblebee 2 stereo camera [11].

\section{Disparity Calculation Approach}

The disparity calculation method described in this paper involves a multi-step process to develop and refine a disparity calculation, specifically in realworld navigational environments.

We have two input pixel images, $I_{L}$ and $I_{R}$, corresponding to the left and right images in a stereo pair, respectively. We assume that $I_{L}$ and $I_{R}$ have been rectified prior to beginning our process. Additionally, we assume that there is a known maximum pixel disparity value present in the image max_disp. This value does not have to be the true maximum disparity between $I_{R}$ and $I_{L}$, but poor choice will either limit the disparity estimation or unnecessarily increase the search space.

In order to maintain positive disparity values, we use $I_{R}$ as our reference image, and $I_{L}$ as our mat- 
ching image. Disparities are measured as the number of pixels in the y direction an object imaged at a pixel in $I_{R}$ moves when observed in $I_{L}$.

\subsection{Generating an Oversegmentation}

Oversegment generation is an important part of the Informative Image Partitioning process, as it defines the finest scale that the image will be represented in. As detailed in [1], the oversegmentation method used strongly affects the partitioning generated.

While simple colour segmentation methods, such as the popular mean shift algorithm, are frequently used in stereo disparity methods, they are not well suited to our task, as they do not produce consistently sized oversegments. Additionally, it is quite possible for objects with a consistent colour to have non-planar disparity behaviour. In this paper, we use the superpixel algorithm defined by [12] to generate 1000 fine-scale partitions over our image.

An oversegmentation needs only be found for our reference image, $I_{R}$, as our technique does not involve matching pre-defined partitions from the reference image to partitions in the matching image.

\subsection{Informative Image Partitioning}

Informative Image Partitioning [1] is an image segmentation technique which incorporates user defined 'interestingness' into the construction of an image segmentation. The method works by merging together oversegments in 'uninteresting' areas while preserving the initial oversegmentation in 'interesting' areas. It does this by taking a set of user input in the form of instructions, either instructing oversegments to merge into one larger segment or explicitly prohibiting two oversegments from merging. From this user input, the method infers a model which describes the action to be taken between any two oversegments in the image, resulting in a multi-scale partitioning across the image. Models trained in this way are able to be applied on not only the image they are trained on, but also other images with similar contents. Images partitioned using this process are shown in Figure 1.

In order to extract the extent of planar surfaces present in the image, we conduct an informative image partitioning in which large, planar regions are defined as uninteresting objects, while nonplanar surfaces are defined as interesting. Generating an informative image partitioning of this type requires user input to determine which elements are planar. For this paper, we have trained a specific model for each image, but the authors of [1] indicate that it is possible to train a model for a class of images, which would largely eliminate the need for human intervention when generating an

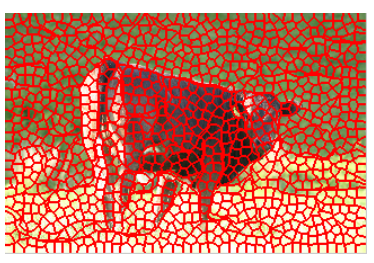

(a)

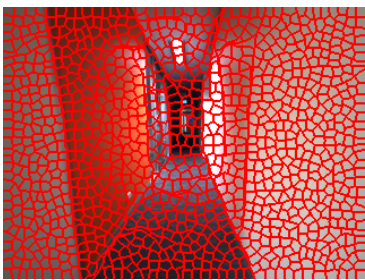

(c)

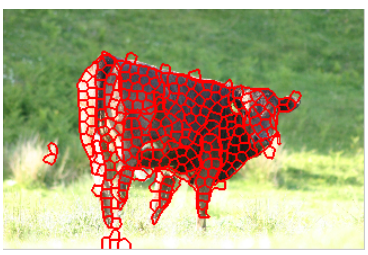

(b)

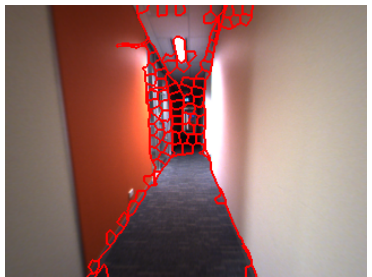

(d)
Figure 1: (a) An oversegmented image from the MSRC data set [13]. (b) The result of applying Informative Image Partitioning to (a). Note that the visually different foreground and background grass are successfully merged. (d) The result of IIP on (c). The orange and white coplanar areas on the left have been successfully merged to a single partition.

image partitioning for navigation. An informative image partitioning is only generated for $I_{R}$.

Following the partitioning process, we have a set of non-overlapping partitions which cover the entire image, $\mathbf{P}$. Each partition $P_{a} \in \mathbf{P}$ is defined by the set of pixel coordinates $(\mathbf{x}, \mathbf{y})_{P_{a}}$ it contains.

\subsection{Coarse Disparity Calculation}

In order to limit the number of labels that we need to optimise in later stages, we wish to generate a baseline disparity for each partition. Effects of occlusions are small when the size of the partition is significantly larger than the occluded portion of the partition. As each partition represents a relatively large image patch, even at the finest scale, we can use a simple statistical correlation method to find a disparity for the partition.

We use the Normalised Cross Correlation between a partition $P_{a}$ in $I_{R}$ and a set of candidate regions in $I_{L}$ containing pixels $(\mathbf{x}, \mathbf{y}+\beta)_{P_{a}}, \beta \in$ $\{0,1, \ldots$, max_disp $\}$ to find a coarse disparity for each partition.

Formally, we find a coarse disparity value $D_{P_{a}}$ as follows:

$$
\begin{aligned}
& D_{P_{a}}=\underset{\beta}{\operatorname{argmax}}\left[\frac{1}{3(n-1)} .\right. \\
& \left.\sum_{(x, y)_{P_{a}}, c} \frac{\left(I_{R}(\mathbf{x}, \mathbf{y}, c)-\bar{P}_{a}\right)\left(I_{L}(\mathbf{x}, \mathbf{y}+\beta, c)-\bar{P}_{a}^{\prime}\right)}{\sigma_{P_{a}} \sigma_{P_{a}^{\prime}}}\right]
\end{aligned}
$$


where $\bar{P}_{a}^{\prime}$ is the mean intensity of the partition at location $(\mathbf{x}, \mathbf{y}+\beta)_{P_{a}}$ in $I_{L}, \sigma_{P_{a}^{\prime}}$ the intensity standard deviation within the same region and $n$ the number of pixels in partition $P_{a} . \quad c$ is a parameter which defines the number of colour intensity channels used. In this paper, we use the RGB intensity space. Figures 4 and 5 (c) show the coarse disparity found in example images.

\subsection{Per-partition Graph-Cut Refinement}

Within each partition, we search for a refinement to the simple per-partition disparity value found above by performing a multi-label graph cut on the values inside each partition $[14,15,16]$.

To do this, we define a graph $\mathcal{G}_{P_{a}}=\left(\mathcal{V}_{P_{a}}, \mathcal{E}_{P_{a}}\right)$, with vertices $\mathcal{V}_{P_{a}}$ corresponding to the pixels contained in partition $i$, and edges $\mathcal{E}_{P_{a}}$ between vertices that are 4 -connected in the image space.

Each vertex within $\mathcal{V}_{P_{a}}$ can take a range of values, $\alpha$, defined by the maximum disparity within the image and the disparity assigned to the partition, $D_{P_{a}}$. We also allow the pixel to take a null value if there is no pixel within range with a high correspondence. For a given partition, the label space is defined as $\left.\alpha \in\left\{-D_{P_{a}}\right),-D_{P_{a}}\right)+1, \ldots,\left(\right.$ max $_{-} d i s p-$ $\left.\left.D_{P_{a}}\right), N U L L\right\}$. We allow for negative disparity values here to allow pixels within a partition to have a lower disparity than $D_{P_{a}}$. These labels reflect a refinement to the coarse estimate calculated above, rather than an absolute disparity.

We then use the $\alpha$-expansion graph-cut algorithm to infer the minimum energy of the equation:

$$
\begin{aligned}
& U\left(\alpha ;\left(\mathbf{x}_{\mathbf{i}}, \mathbf{y}_{\mathbf{i}}\right)\right)=\lambda \sum_{i \in \mathcal{V}_{P_{a}}} U_{1}\left(\alpha_{i} ;\left(x_{i}, y_{i}\right)\right) \\
& +\sum_{i j \in \mathcal{E}_{P_{a}}} U_{2}\left(\left(x_{i}, y_{i}\right) ;\left(x_{j}, y_{j}\right)\right) \delta\left[\left(\alpha_{i} \neq \alpha_{j}\right), \alpha_{i, j} \neq N U L L\right.
\end{aligned}
$$

In which the data term $U_{1}$ represents the cost of assigning vertex $i$ to a particular disparity level. The local smoothing term $U_{2}$ represents the cost associated with assigning different labels to adjacent nodes. We relax the smoothness constraint between nodes assigned to null disparity and other nodes.

The data term $U_{1}$ is given by the average intensity difference between the RGB pixel at $\left(x_{i}, y_{i}\right)$ in $I_{R}$ and the RGB pixel at the location in $I_{L}$ at $(x, y+$ $\alpha)$.

$$
\begin{aligned}
& U_{1}\left(\alpha_{i} \neq N U L L ;\left(x_{i}, y_{i}\right)\right)= \\
& \left\{\begin{array}{l}
\infty, \quad y_{i}+\alpha_{i}+D_{P_{a}}<y_{i} \\
\left|I_{R}\left(x_{i}, y_{i}\right), I_{L}\left(x_{i},\left(y_{i}+\alpha_{i}+D_{P_{a}}\right)\right)\right|_{2}, \text { otherwise }
\end{array}\right.
\end{aligned}
$$

The data term value for the special case $\alpha=N U L L$ is derived from the minimum data term value at all other values of alpha:

$$
\begin{aligned}
& U_{1}\left(\alpha_{i}=N U L L ;\left(x_{i}, y_{i}\right)\right)= \\
& 1-\min \left[U_{1}\left(\alpha_{i} \neq N U L L ;\left(x_{i}, y_{i}\right)\right]\right.
\end{aligned}
$$

We use a simple Potts model smoothing term for $U_{2}$ with a relatively low smoothing constant $\lambda$, as we do not want to over smooth the disparity results within the partition.

$$
U_{2}\left(\left(x_{i}, y_{i}\right) ;\left(x_{j}, y_{j}\right)\right)=\lambda\left|I_{R}\left(x_{i}, y_{i}\right), I_{R}\left(x_{j}, y_{j}\right)\right|_{2}
$$

For very large planar regions, the smoothing term becomes less important, as the robust plane fitting technique can itself reduce the effect of outliers. We make use of Shai Bagon's matlab wrapper for the graph cut algorithm [17] to perform inference.

\subsection{Per-partition Plane Fitting}

We want to fit a plane model $d=a x+b y+c$ to the disparity values calculated above, where $a, b$ and $c$ are plane parameters and $\mathrm{d}$ is the corresponding disparity at pixel $(x, y)$. We fit this plane using the well-known least squares approach [18].

Once we have a plane fitted to the data, we check to ensure that disparities assigned to pixels within the partition by the fitted plane do not violate obvious constraints on the disparity. Clearly, it is not possible for the disparity at any point in the image to be less than zero. Similarly, disparity values greater than the defined maximum max_disp violate our initial assumptions, and should not be included.

If a fitted plane violates either of these constraints, we assume that the patch is significantly non-planar and use the disparity estimates obtained from the graph cut directly for regions of the patch that violate our assumptions.

\subsection{Generating a Complete Disparity Map}

The complete disparity map is generated in a piecewise fashion by applying the steps described in sections 3.3 to 3.5 to each partition in the image. This approach can lead to irregularities at the boundaries of partitions, which results in a 'mosaic' appearance in the disparity map. As this effect is most pronounced at boundaries of small segments, and may be the result of actual disparity edges in the image, we do not apply any additional smoothing to reduce this effect. 


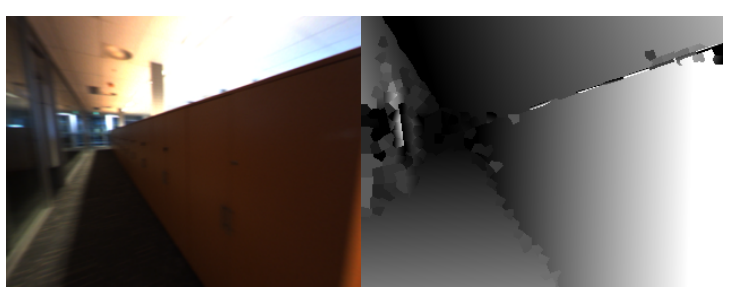

(a)

(b)

Figure 2: (a) Sample input image from our office environment data set. (b) Calculated disparity map for (a). Disparity values have been scaled for clarity.

\section{Results}

Our approach aims to produce disparity maps to assist in navigation, making the commonly used Middlebury disparity test set impractical for estimation of the true performance of our algorithm. To test our approach in the context for which it has been developed, we have captured a set of realworld scenes using a Bumblebee 2 stereo camera to test our disparity calculation algorithm.

\subsection{Office Environment Scenes}

Indoor scenes in an office environment consist largely of planar surfaces, such as floors, walls, desks and doors. Non-planar objects within an office are typically relatively small, exhibiting little change of disparity within the object. This indicates that a single disparity value will be sufficient to indicate the relative distance between the observer and the object.

Five images have been taken along various corridors within the research lab under normal, daytime lighting conditions. In several cases, glass walls are also present in the image. Figure 4 illustrates a step-by-step walkthrough of our algorithm, along with the results of three competing stereo disparity calculation methods on an image from our indoor data set. Figure 2 shows the result of our disparity calculation algorithm on two additional images from this data set.

\subsection{Urban Scenes}

Outdoor scenes in an urban environment also consist of a large number of planar or near-planar objects, such as roads, pavement, walls, advertising boards and fencing. Non-planar objects in outdoor scenes, such as other pedestrians, trees, lampposts and vehicles, are generally larger than those in an indoor environment, and thus will be represented by multiple partitions within our framework. The disparity behaviour of such objects can be well represented by faceted disparity planes.

We have a set of eight images taken on local streets (a)

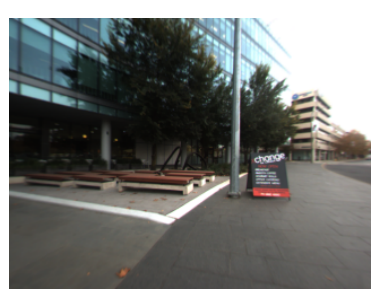

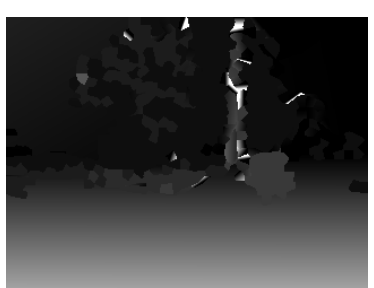

(b)
Figure 3: (a) Sample input image from our street scene data set. (b) Calculated disparity map for (a). Disparity values have been scaled for clarity.

in daylight conditions, with a variety of challenging buildings. Figure 5 shows the performance of several disparity calculation methods, including ours, on a sample scene from this data set. Figure 3 shows the disparity maps generated by our method on two additional images from this data set.

\subsection{Discussion}

The results in both indoor and outdoor scenes are quite good, particularly in the ground plane. Unfortunately, the IIP process does not perfectly segment planar regions, resulting in some regions with undesired segmentation and incorrect disparity values. While the method is good at finding appropriate disparity values in weakly textured areas, in some cases errors in the per-plane disparity calculation (Section 3.4) strongly affect the parameters of the plane, resulting in a skewed distribution from plane fitting. Despite these problems, the disparity maps we generate outperform benchmark methods $[4,3]$.

\section{Conclusions}

By segmenting the image into regions which follow a planar disparity distribution, we are able to produce qualitatively improved disparity estimates in low-textured planar regions, which pose problems for traditional disparity calculation methods. We show that our approach produces good stereo disparity estimates in a variety of challenging real-world environments, both indoor and outdoor. This supports the use of IIP segmentation and the assumption of an underlying planar disparity distribution when generating disparity maps. We demonstrate that our method qualitatively outperform benchmark methods on real-world data.

\subsection{Future Work}

The performance of our method on small segments is not better than some state-of-the-art techniques. Given that small segments are generally non-planar, it is possible to apply an alternate, non planefitting disparity calculation method in these re- 
gions to improve the corresponding disparity estimate.

In this paper we use only intensity information to determine disparity. Use of texture or other more sophisticated features, such as the DAISY descriptor [19] would improve matching accuracy and thus disparity calculation performance.

Additionally, we use a simple outlier elimination method in plane fitting. Use of a RANSAC method [20] would more robustly fit planes to our data, potentially improving the result.

Finally, use of an inter-segment smoothing method to reduce the mosaic appearance of the final disparity map, would potentially eliminate false disparity edges in our results.

\section{References}

[1] Brewer, N., Liu, N., Wang, L.: Guided informative image partitioning. In: Joint IAPR International Workshops on Structural and Syntactic Pattern Recognition, Cesme, Turkey (2010)

[2] Scharstein, D., Szeliski, R.: A taxonomy and evaluation of dense two-frame stereo correspondence algorithms. International Journal of Computer Vision 47 (2001) 7-42

[3] Boykov, Y., Veksler, O., Zabih, R.: Markov random fields with efficient approximations. In: CVPR '98: Proceedings of the IEEE Computer Society Conference on Computer Vision and Pattern Recognition, Washington, DC, USA, IEEE Computer Society (1998) 648

[4] Kolmogorov, V., Zabih, R.: Computing visual correspondence with occlusions via graph cuts. In: In International Conference on Computer Vision. (2001) 508-515

[5] Hong, L., Chen, G.: Segment-based stereo matching using graph cuts. Computer Vision and Pattern Recognition, IEEE Computer Society Conference on 1 (2004) 74-81

[6] Bleyer, M., Gelautz, M.: Graph-cut-based stereo matching using image segmentation with symmetrical treatment of occlusions. Image Commun. 22 (2007) 127-143

[7] Yang, Q., Engels, C., Akbarzadeh, A.: Near real-time stereo for weakly-textured scenes. (2008) xx-yy

[8] Wei, Y., Quan, L.: Region-based progressive stereo matching. In: In Conference on Computer Vision and Pattern Recognition. (2004) 106-113
[9] Fukunaga, K., Hostetler, L.: The estimation of the gradient of a density function, with applications in pattern recognition. 21 (1975) $32-40$

[10] Woodford, O., Torr, P., Reid, I., Fitzgibbon, A.: Global stereo reconstruction under second-order smoothness priors. 31 (2009) 2115-2128

[11] Research, P.G.: Bumblebee stereo vision camera systems. (2009)

[12] Mori, G.: Guiding model search using segmentation. ICCV 2005. 2 (2005) 1417-1423 Vol. 2

[13] Shotton, J., Winn, J., Rother, C., Criminisi, A.: Textonboost: Joint appearance, shape and context modeling for multi-class object recognition and segmentation. In: ECCV. (2006) 1-15

[14] Boykov, Y., Veksler, O., Zabih, R.: Fast approximate energy minimization via graph cuts. IEEE Trans. Pattern Anal. Mach. Intell. 23 (2001) 1222-1239

[15] Kolmogorov, V., Zabih, R.: What energy functions can be minimized via graph cuts? In: ECCV '02: Proceedings of the 7th European Conference on Computer Vision-Part III, London, UK, Springer-Verlag (2002) 6581

[16] Boykov, Y., Kolmogorov, V.: An experimental comparison of min-cut/max-flow algorithms for energy minimization in vision. IEEE Trans. Pattern Anal. Mach. Intell. 26 (2004) 1124-1137

[17] Bagon, S.: Matlab wrapper for graph cut. (2006)

[18] Bishop, C.M.: Pattern Recognition and Machine Learning (Information Science and Statistics). Springer-Verlag New York, Inc., Secaucus, NJ, USA (2006)

[19] Tola, E., Lepetit, V., Fua, P.: A fast local descriptor for dense matching. In: Conference on Computer Vision and Pattern Recognition, Alaska, USA (2008)

[20] Fischler, M.A., Bolles, R.C.: Random sample consensus: a paradigm for model fitting with applications to image analysis and automated cartography. Commun. ACM 24 (1981) 381395 


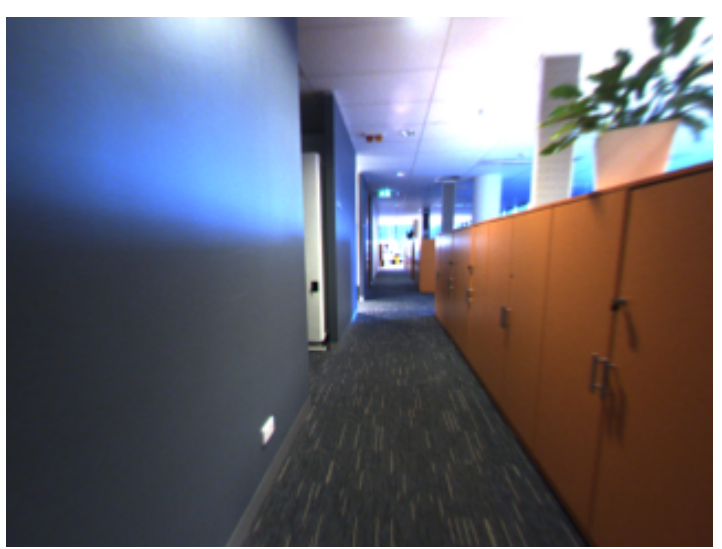

(a) Input image $I_{R}$

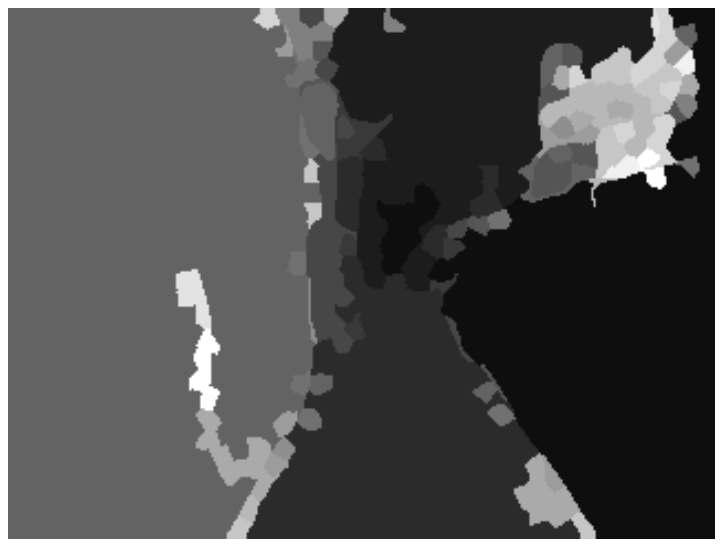

(c) NCC disparity results for each segment (Subsection 3.3)

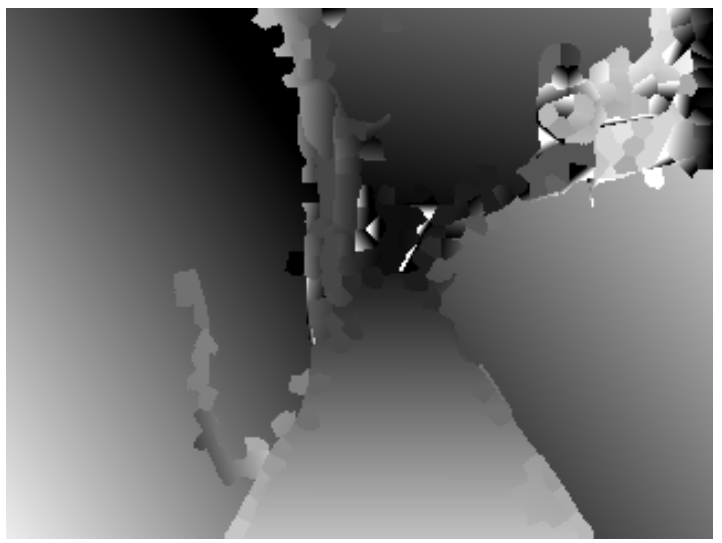

(e) Full disparity map generated using our method (Subsection 3.5)

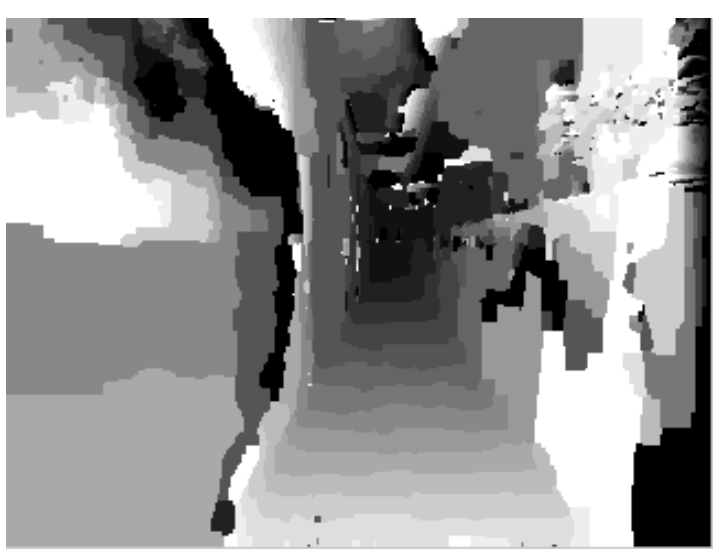

(g) Disparity map generated using [4]

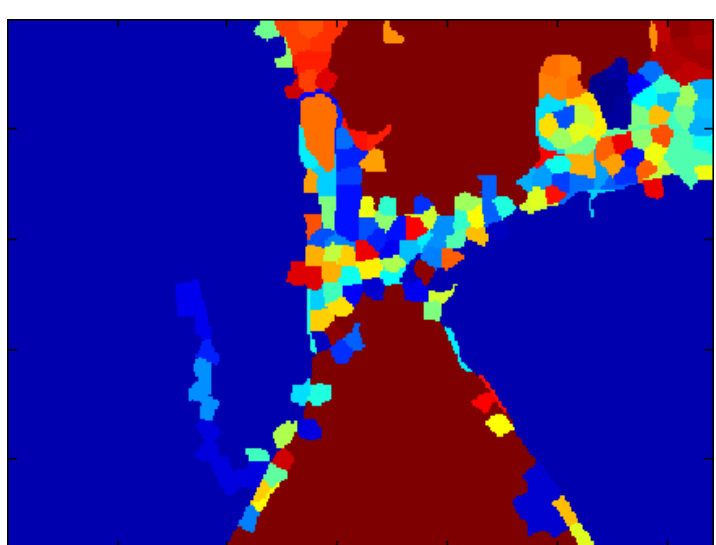

(b) Segments generated using IIP [1]

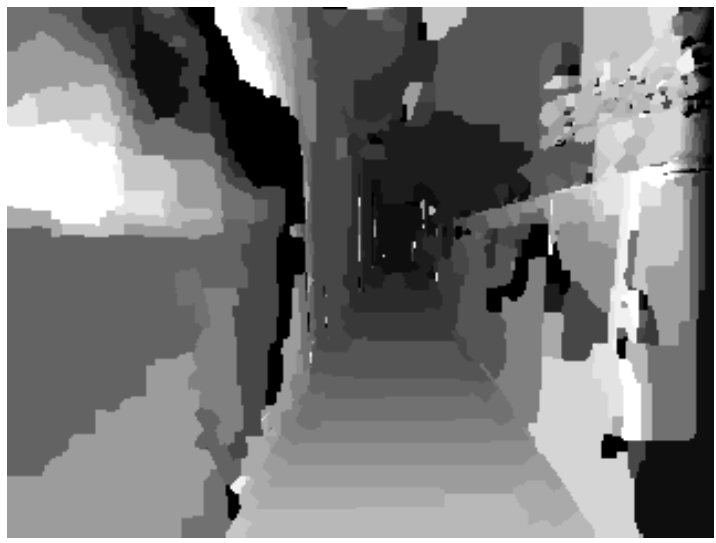

(d) Graph Cut disparity results for each segment (Subsection 3.4)

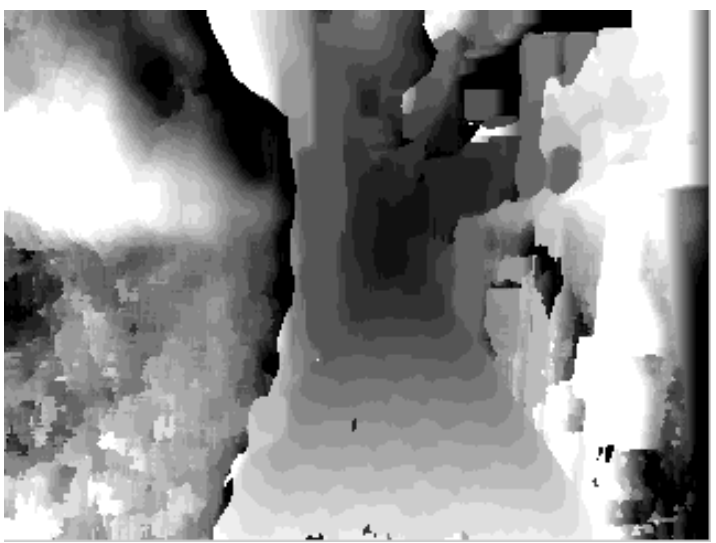

(f) Disparity map generated using stereo correlation

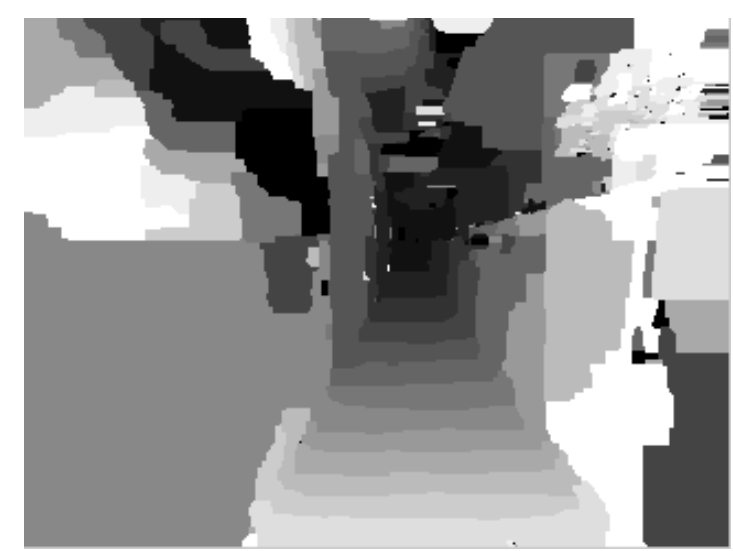

(h) Disparity map generated using [3]

Figure 4: The proposed disparity estimation process applied to a typical scene from within an office environment captured with a Bumblebee 2 stereo camera. Our method performs significantly better on the weakly textured walls and ceiling than benchmark methods. 


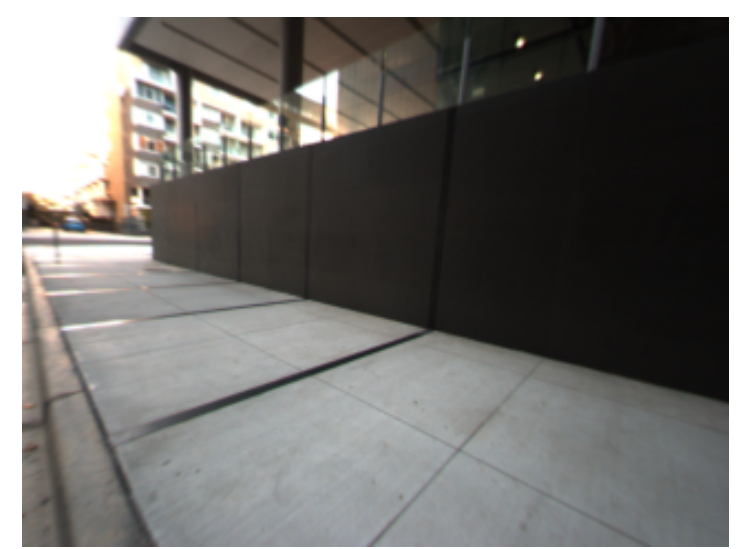

(a) Input image $I_{R}$

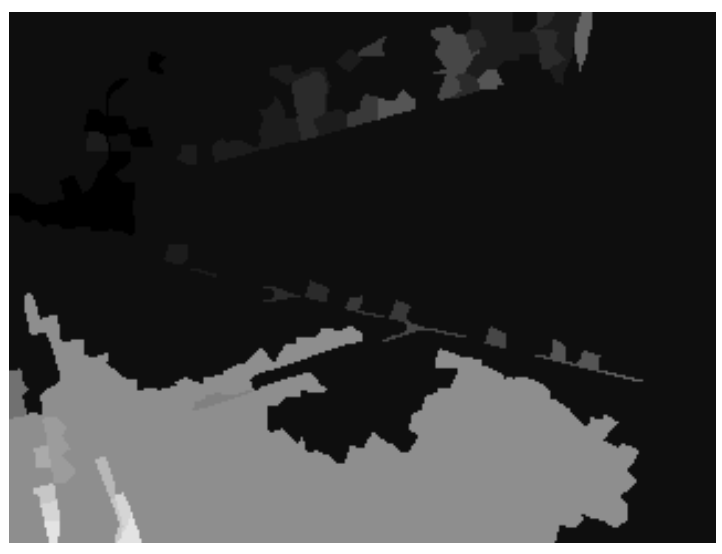

(c) NCC disparity results for each segment (Subsection $3.3)$

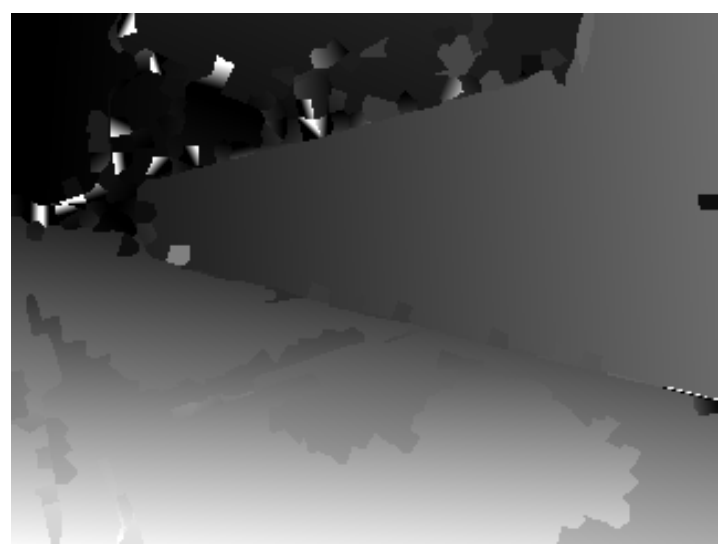

(e) Full disparity map generated using our method (Subsection 3.5)

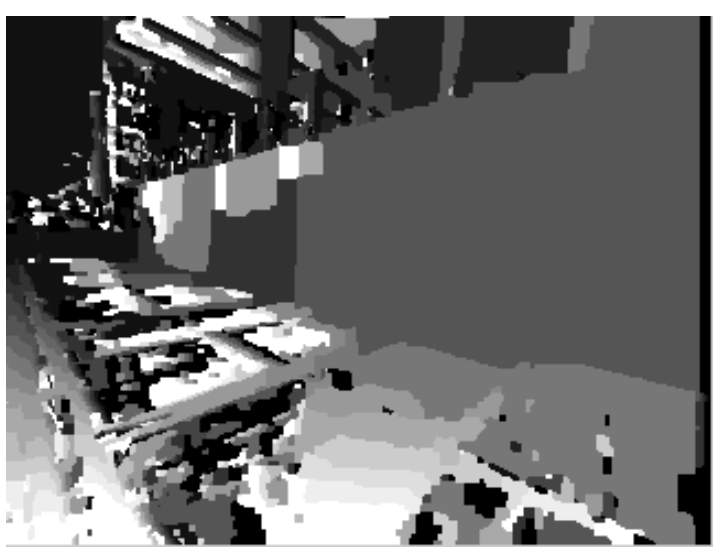

(g) Disparity map generated using [4]

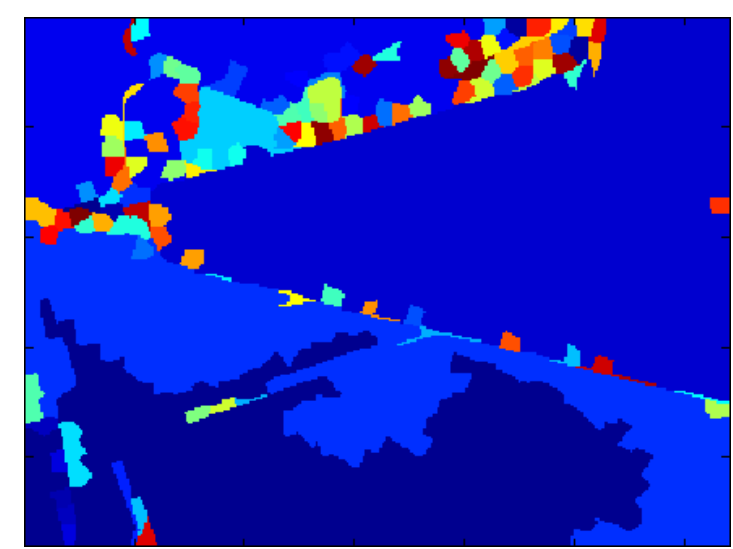

(b) Segments generated using IIP [1]

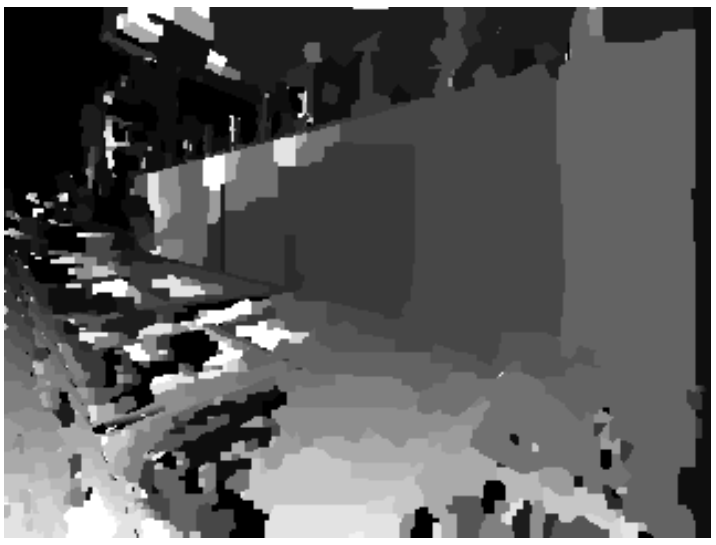

(d) Graph Cut disparity results for each segment (Subsection 3.4)

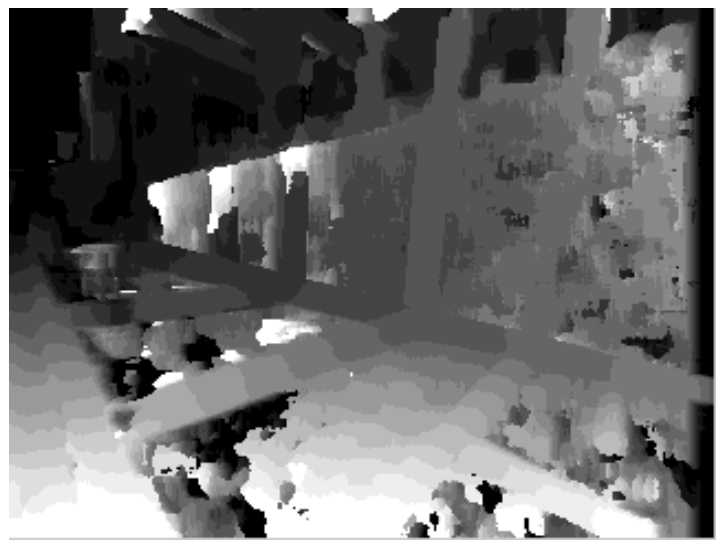

(f) Disparity map generated using stereo correlation

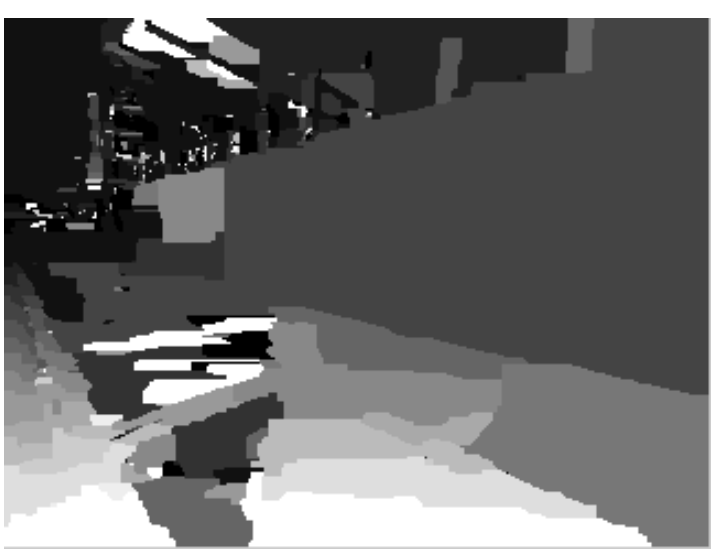

(h) Disparity map generated using [3]

Figure 5: An outdoor image captured with a Bumblebee 2 stereo camera under normal, daytime conditions. Performance of our method is visually excellent, particularly in the weakly textured road and path regions, and along the adjacent wall. 